\title{
线性模型中误差方差估计的正态逼近的 非一致性界限
}

\author{
赵林城白志东 \\ (中国科技大学数学系, 合肥)
}

\section{畝要}

在一般的线性模型 $Y_{j}=x_{j}^{\prime} \beta+e_{j}(j=1, \cdots, n, \cdots)$ 中，根据观测值 $Y_{1}, \cdots$ ， $Y_{n}$, 可以得出误差方差 $\sigma^{2}$ 的基于残差平方和的估计 $\hat{\sigma}_{n}^{2}$. 分别用 $F_{n}$ 和 $\Phi$ 记 r.v. ( $\hat{\sigma}_{n}^{2}$ $\left.-\sigma^{2}\right) / \sqrt{\operatorname{Var}\left(\hat{\sigma}_{n}^{2}\right)}$ 和 $N(0,1)$ 的分布函数. 本文证明了, 若 $\left\{e_{i}\right\}$ 独立, 且存在常数 $D_{1}, D_{2}$, 使

$$
\frac{1}{n} \sum_{k=1}^{n} E\left(e_{k}^{6}\right) \leqslant D_{1}<\infty, \frac{1}{n} \sum_{k=1}^{n} \operatorname{Var}\left(e_{k}^{2}\right) \geqslant D_{2}>0,
$$

则存在与 $n$ 及 $x$ 都无关的常数 $c$, 使对所有 $n$ 和 $x$, 有

$$
\left|F_{n}(x)-\Phi(x)\right| \leqslant C /\left[\sqrt{n}(1+|x|)^{3}\right] \text {. }
$$

这一结果对文献 [1] 中的猜测给出了肯定的回答, 且正如文献 [2]中所指出的, 它是 一个不可改进的理想结果。

\section{一、引言和主要结果}

考虑线性模型

$$
Y_{i}=x_{j}^{\prime} \beta+e_{j}, j=1, \cdots, n, \cdots,
$$

此处 $\left\{x_{i}\right\}$ 是已知的 $p$ 维设计点列, $\beta$ 为末知的 $P$ 维回归系数向量, $\left\{e_{j}\right\}$ 为随机误差序列, 满足 下述条件:

$$
\left\{\begin{array}{l}
e_{1}, e_{2}, \cdots \text { 独立, } \\
E\left(e_{i}\right)=0,0<E\left(e_{i}^{2}\right)=\sigma^{2}<\infty, j=1, \cdots, n, \cdots
\end{array}\right.
$$

误差方差 $\sigma^{2}$ 是模型 (1) 的重要参数. 根据观测值 $Y_{1}, \cdots, Y_{n}$, 可以得出 $\sigma^{2}$ 的基于残差平方 和的无偏估计 $\hat{\sigma}_{n}^{2}$ :

$$
\hat{\sigma}_{n}^{2}=\frac{1}{n-r_{n}}\left\{\sum_{k=1}^{n} e_{k}^{2}-\sum_{j=1}^{n}\left(\sum_{k=1}^{n} a_{n j k} e_{k}\right)^{2}\right\},
$$

其中, $\left\{a_{n j k}\right\}$ 为由设计矩阵 $X_{n}=\left(x_{1} \vdots \cdots \vdots x_{n}\right)$ 决定的一组实数, 满足

$$
\sum_{k=1}^{n} a_{n j k} a_{n l k}= \begin{cases}1, & \text { 若 } j=l, \\ 0, & \text { 若 } j \neq l .\end{cases}
$$

本文 1982 年 7 月 8 日收到. 
当 $n$ 充分文时, $r_{n}=\operatorname{rank}\left(X_{n}\right)$ 稳定到某个非负整数 $r \leqslant p$, 故以下记 $r_{n}$ 为 $r$.

分别以 $F_{n}$ 和 $\Phi$ 记 $Q_{n}=\left(\hat{\sigma}_{n}^{2}-\sigma^{2}\right) / \sqrt{\operatorname{Var}\left(\hat{\sigma}_{n}^{2}\right)}$ 和 $N(0,1)$ 的分布函数. 赵林城、陈希 請 ${ }^{[1]}$ 证得, 若 $e_{1}, e_{2}, \cdots$ iid., $\operatorname{Var}\left(e_{1}^{2}\right)>0$ 且 $E\left(e_{1}^{6}\right)<\infty$, 则存在与 $n$ 及 $x$ 都无关的常数 $C>0$, 使对所有 $n$ 和 $x$, 有

$$
\left|F_{n}(x)-\Phi(x)\right| \leqslant C /\left[\sqrt{n}(1+|x|)^{3}\right] .
$$

最近, 苏淳 ${ }^{[2]}$ 指出, 正态逼近的这个非一致性界限已是不可改进的了.

赵和陈在文献 [1] 中猜测, 上述结果可以推广到 $\left\{e_{j}\right\}$ 独立而不必同分布的更一般的情 形. 本文证实了这一猜测, 换句话说, 我们证明了下述定理:

定理 1. 设在线性模型 (1) 之下 (2) 式成立, 且存在常数 $D_{1}, D_{2}$, 使

$$
\begin{gathered}
\frac{1}{n} \sum_{k=1}^{n} E\left(e_{k}^{6}\right) \leqslant D_{1}<\infty, \\
\frac{1}{n} \sum_{k=1}^{n} d_{k}^{2} \geqslant D_{2}>0, \quad d_{k}^{2}=\operatorname{Var}\left(e_{k}^{2}\right)=E\left(e_{k}^{4}\right)-\sigma^{4},
\end{gathered}
$$

则 (4) 式仍成立.

\section{二、若干引理}

定理 1 的证明很复杂,因此将其主要部分分解为一些引理. 为书写简便,特作以下约定:

1. 用 $C$ 记一与 $n$ 及 $x$ 都无关的常数,每次出现,即使在同一式内,都可取不同之值.

2. 文中各处出现的 $\eta>0, \mu>0, \lambda>0$ 均可事先取成相同的, 均为与 $n$ 及 $x$ 都无关的 常数. 又, “当 $n$ 充分大”一语常常省去, 望不致引起误解.

3. 设 $g_{1}(t), g_{2}(t)$ 为定义于 $R^{1}$ 的复值函数(可与 $n$ 有关). 称

$$
g_{1} \sim g_{2},
$$

若 $\exists \eta>0$, 使

$$
\int_{|z|<\sqrt{n} \eta}|t|^{-1}\left|g_{1}(t)-g_{2}(t)\right| d t \leqslant C / \sqrt{n} .
$$

又, 集 $A$ 的指示函数记为 $I(A)$, 集 $A$ 中元素个数记为\#(A). 在本文中, $i$ 总用来记 $\sqrt{-1}$

记

$$
\begin{aligned}
& \Lambda_{n}=\{1,2, \cdots, n\}, \quad \lambda_{k}=\sum_{j=1}^{r_{1}} a_{n k}^{2}, \\
& \Lambda_{n 0}=\left\{k: 1 \leqslant k \leqslant n, \lambda_{k}>1 / 4\right\},
\end{aligned}
$$

财由 $\sum_{k=1}^{n} \lambda_{k}=r$, 知\# $\left(\Lambda_{n 0}\right)<4 r$. 令

$$
\begin{gathered}
\hat{e}_{k}=e_{k} I\left(\left|e_{k}\right| \leqslant n^{1 / 4}\right), \quad \xi_{k}=\hat{e}_{k}^{2}-E\left(\hat{e}_{k}^{2}\right), \\
\zeta_{k}= \begin{cases}\left(1-4 \lambda_{k}\right)\left(\hat{e}_{k}^{2}-E \hat{e}_{k}^{2}\right), & \text { 当 } k \in \Lambda_{n}-\Lambda_{n 0}, \\
(1-4 r)\left(e_{k}^{2}-\sigma^{2}\right), & \text { 当 } k \in \Lambda_{n 0 .} .\end{cases} \\
\sigma_{n k}^{2}=E \zeta_{k}^{2}, \quad \alpha_{n k}-E\left|\zeta_{k}\right|^{3}, \quad \text { 当 } k \in A_{n} .
\end{gathered}
$$




$$
n B_{n}^{2}=\sum_{k=1}^{n} d_{k}^{2}, \quad n \widetilde{B}_{n}^{2}=\sum_{k=1}^{n} \sigma_{n k}^{2}, \quad n \alpha_{n}=\sum_{k=1}^{n} \alpha_{n k}
$$

注意, $\lambda_{k}, \hat{\varepsilon}_{k}, \xi_{k}, \zeta_{k}$ 等都与 $n$ 有关.

引理 1. 存在与 $n$ 无关的常数 $D_{3}>0, D_{i}^{0}>0, b_{i}>0, j=1,2,3$, 使

$$
B_{n}^{2} \leqslant D_{3}, \quad\left|B_{n}^{2}-\widetilde{B}_{n}^{2}\right| \leqslant C / \sqrt{n}, \quad \widetilde{B}_{n}^{2} \geqslant D_{2}^{\alpha}, \quad \alpha_{n} \leqslant D_{1}^{0},
$$

且集合

$$
A_{n}=\left\{k: \sigma_{n k}^{2} \geqslant b_{2}, \alpha_{n k} \leqslant b_{3}, 1 \leqslant k \leqslant n\right\}
$$

的元素个数\# $\left(A_{n}\right) \geqslant b_{1} n$.

存在与 $n,\left\{a_{n j k}\right\}$ 无关的常数 $L>0$, 使集合

$$
D_{n}=\left\{k: 1 \leqslant k \leqslant n, a_{n j k}^{2} \leqslant L / n \text { 对 } j=1, \cdots, r\right\}
$$

与集合 $A_{n}$ 之交的元素个数\# $\left(A_{n} D_{n}\right) \geqslant \frac{1}{2} b_{1} n$.

证. 只须证明 $\left|B_{n}^{2}-\widetilde{B}_{n}^{2}\right| \leqslant C / \sqrt{n}$, 其余各式的证明见文献 [3] 中引理 1, 引理 7.分 别以 $\Sigma_{(0)}, \Sigma^{\prime}$ 记对 $A_{n 0}$ 及 $\Lambda_{n}-\Lambda_{n 0}$ 中的足标求和. 由

$$
0 \leqslant \lambda_{k} \leqslant 1, \quad \sum_{k=1}^{n} \lambda_{k}=r
$$

及（5) 式,有

$$
\begin{aligned}
\left|n B_{n}^{2}-n \widetilde{B}_{n}^{2}\right| \leqslant & 2 \sum_{(0)}\left(8 r+16 r^{2}\right) E e_{k}^{4}+\Sigma^{\prime}\left(8 \lambda_{k}+16 \lambda_{k}^{2}\right) E \hat{e}_{k}^{4} \\
& +\sum^{\prime}\left(E e_{k}^{4}-E e_{k}^{4}\right)+\Sigma^{\prime}\left[\sigma^{4}-E^{2}\left(e_{k}^{2}\right)\right] \\
\leqslant & 48 r^{2} \sum_{\left.{ }_{0}\right)} E e_{k}^{4}+24 \Sigma^{\prime} \lambda_{k} E e_{k}^{4} \\
& +\sum^{\prime} E e_{k}^{4} I\left(e_{k}^{2}>\sqrt{n}\right)+2 \sigma^{2} \Sigma^{\prime} E e_{k}^{2} I\left(e_{k}^{2}>\sqrt{n}\right) \\
\leqslant & 48 r^{2} \sum_{(0)} \sqrt{\sigma^{2} E e_{k}^{6}}+24 \Sigma^{\prime} \lambda_{k} \sqrt{\sigma^{2} E e_{k}^{n}}+\frac{1}{\sqrt{n}} \Sigma^{\prime} E e_{k}^{6}+\frac{2 \sigma^{2}}{n} \Sigma^{\prime} E e_{k}^{6} \\
\leqslant & 48 r^{2} \sigma \sum_{(0)} \sqrt{D_{1} n}+24 \sigma\left(\Sigma^{\prime} \lambda_{k}^{2} \Sigma^{\prime} E e_{k}^{6}\right)^{1 / 2}+\sqrt{n} D_{1}+2 \sigma^{2} D_{1} \\
\leqslant & C \sqrt{n},
\end{aligned}
$$

这就是所要证明的.

引理 2. 以 $\varphi_{n k}(t)$ 记 $\zeta_{k}$ 的 c. f., 则在定理条件下, $\exists \eta, \lambda$, 使对所有 $k \in A_{n}$, 有

$$
\left|\varphi_{n k}\left(t / \sqrt{n} \widetilde{B}_{n}\right)\right| \leqslant \exp \left(-\lambda t^{2} / n\right) \text {, 当 }|t| \leqslant \sqrt{n} \eta \text {. }
$$

证. 见文献 [3], 引理 2 .

由引理 1, 我们可在 $A_{n} D_{n}-\Lambda_{n 0}$ 中取出 [ $n^{7 / 8}$ ] 个元素,组成集合 $\Lambda_{n 3}$. 将 $\Lambda_{n}-\Lambda_{n 0}$ 一 $\Lambda_{n 1}$ 分成 $\Lambda_{n 1}, \Lambda_{n 2}$ 两个集合,使 $\#\left(\Lambda_{n ;} A_{n}\right) \geqslant b_{1} n / 3, j=1,2$. 记

$$
\begin{gathered}
S_{n}=\frac{1}{\sqrt{n} \widetilde{B}_{n}} \sum \zeta_{k}, \quad S_{n}^{\prime}=\frac{1}{\sqrt{n} \widetilde{B}_{n}} \Sigma^{\prime} \zeta_{k}, \\
S_{n *}=\frac{1}{\sqrt{n} \widetilde{B}_{n}} \sum(*) \zeta_{k}, \quad *=0,1,2,3 .
\end{gathered}
$$




$$
\Delta_{n *}=\frac{1}{\sqrt{n} \widetilde{B}_{n}} \sum_{j=1}^{r} \sum_{k \neq 1} a_{n j k} a_{n i l} e_{k} e_{l}, \quad *=1,2,3 .
$$

$\sum, \Sigma^{\prime}, \sum_{(*)}$ 分别对属于 $\Lambda_{n}, A_{n}-\Lambda_{n 0}$ 及 $\Lambda_{n *}$ 中的指标求和.

引理 3.

$$
\begin{gathered}
E\left|S_{n *}\right|^{m} \leqslant C, *=0,1,2,3 ; m=1,2,3,4 . \\
E\left|\Delta_{n 3}\right|^{4} \leqslant C n^{-5 / 2}, \\
E\left|\Delta_{n 1}\right| \leqslant C / \sqrt{n}, \quad E\left|\Delta_{n 1}\right|^{m} \leqslant C / n, \quad m=2,3,4 . \\
E\left(\left|S_{n 1}^{m}\right| \Delta_{n 1}^{2}\right) \leqslant C / n, \quad m=1,2 . \\
E\left|S_{n 1}^{m} \Delta_{n 1}\right| \leqslant C / \sqrt{n}, \quad m=1,2,3 . \\
E\left|S_{n 1} \Delta_{n 1}^{3}\right| \leqslant C / n .
\end{gathered}
$$

证. 在以下证明中, 凡用一个和号表示的多重求和的范围圴为: 各个足标两两不同且属 于指定的集合 $\Lambda_{n * *}$. (11)式容易由 $\left|\zeta_{k}\right| \leqslant C \sqrt{n}$ 及 $\sum_{(*)} E\left|\zeta_{k}\right|^{3} \leqslant C_{n}$ 得出. 为证 (12) 式, 只需证明: 若 $b_{k}^{2} \leqslant C / n$ 对 $k \in \Lambda_{n 3}$, 则有

$$
E\left(\sum_{(3)} b_{j} b_{k} e_{j} e_{k}\right)^{4} \leqslant C / \sqrt{n} \text {. }
$$

事实上, 由 $\sum E\left(e_{k}^{k}\right) \leqslant C n$ 知

$$
\begin{aligned}
E\left(\sum_{(3)} b, b_{k} e_{i} e_{k}\right)^{4} & \leqslant C\left(\sum_{(3)} b_{k}^{4} E e_{k}^{4}\right)^{2}+C\left(\sum_{(3)} b_{k}^{2} \sigma^{2}\right)^{4} \\
& \leqslant\left(C n^{-2} \cdot C n\right)^{2}+\left(C n^{-1} \cdot n^{7 / 8}\right)^{4} \\
& \leqslant C / \sqrt{n} .
\end{aligned}
$$

为证以下各式,不妨设

$$
\Delta_{n 1}=\frac{1}{\sqrt{n} \tilde{B}_{n}} \sum_{(1)} a_{l} a_{k} e_{1} e_{k},
$$

此处 $\sum_{(1)} a_{k}^{2} \leqslant 1$. 由 $E\left|\Delta_{n 1}\right| \leqslant \sqrt{E\left(\Delta_{n 1}^{2}\right)}$ 及 $E\left|\Delta_{n 1}^{3}\right| \leqslant E \Delta_{n 1}^{2}+E \Delta_{n 1}^{4}$, 知只需对 $m=2$, 4 证明 (13) 式, 今以 $m=4$ 为例, 有

$$
\begin{aligned}
E \Delta_{n 1}^{4} & \leqslant C n^{-2}\left\{\left(\sum_{(1)} a_{k}^{4} E e_{k}^{4}\right)^{2}+\left(\sum_{(1)} a_{k}^{2} \sigma^{2}\right)^{4}\right\} \\
& \leqslant C n^{-2}\left\{\sum_{(1)} a_{k}^{4}\left(E e_{k}^{4}\right)^{2}+C\right\} \leqslant C n^{-2}\left\{\sum_{(1)} a_{k}^{4} \sigma^{2} E e_{k}^{6}+C\right\} \\
& \leqslant C n^{-2}\{C n+C\} \leqslant C / n .
\end{aligned}
$$

由 $\left|S_{n 1} \Delta_{n 1}^{2}\right| \leqslant \Delta_{n 1}^{2}+S_{n 1}^{2} \Delta_{n 1}^{2}$ 知, 为证 (14) 式, 只需对 $m=2$ 证明之. 为此, 记 $Z_{k}=a_{k} e_{k}$, 注 意到

$$
\begin{aligned}
& \sum_{(1)} E Z_{k}^{2} \leqslant C, \quad \sum_{(1)} E \zeta_{k}^{2} Z_{k}^{2} \leqslant C n, \\
& \left(\sum_{(1)} E\left|Z_{k} \zeta_{k}\right|\right)^{2} \leqslant \sum_{(1)} a_{k}^{2} \sum_{(1)}\left(E\left|e_{k} \zeta_{k}\right|\right)^{2} \leqslant \sum_{(1)} \sigma^{2} E \zeta_{k}^{2} \leqslant C n, \\
& \left.\begin{array}{rl}
\left(\sum_{(1)} E\left|Z_{k}^{2} \zeta_{k}\right|\right)^{2} & \leqslant \sum_{(1)} a_{k}^{4} \sum_{(1)}\left(E\left|e_{k}^{2} \zeta_{k}\right|\right)^{2} \leqslant \sum_{(1)} \sigma^{2} E\left(e_{k}^{2} \zeta_{k}^{2}\right) \\
& \leqslant C \sigma^{2} \sum_{(1)} E\left(e_{k}^{6}\right) \leqslant C n .
\end{array}\right\}
\end{aligned}
$$

以及

$$
\left(\sum_{(1)} Z_{i} Z_{k}\right)^{2}=2 \sum_{(1)} Z_{j}^{2} Z_{k}^{2}+4 \sum_{(1)} Z_{j}^{2} Z_{k} Z_{l}+\sum_{(1)} Z_{i} Z_{k} Z_{l} Z_{m},
$$

有

$$
\begin{aligned}
E S_{n 1}^{2} \Delta_{n 1}^{2} \leqslant & C n^{-2}\left\{\sum_{(1)} E\left(\zeta_{j}^{2} Z_{j}^{2}\right) E\left(Z_{k}^{2}\right)+\sum_{(1)} E \zeta_{j}^{2} E Z_{k}^{2} E Z_{l}^{2}\right. \\
& \left.+\sum_{(1)} E\left|\zeta_{j} Z_{j}^{2}\right| E\left|\zeta_{k} Z_{k}^{2}\right|+\sum_{(1)} E Z_{j}^{2} E\left|Z_{k} \zeta_{k}\right| E\left|Z_{l} \zeta_{l}\right|\right\} \\
\leqslant & C n^{-2}\left\{C n+C_{n}+C_{n}+C_{n}\right\} \leqslant C / n .
\end{aligned}
$$


这就证明了（14）式. (15) 式由 $E\left|S_{n 1}^{3} \Delta_{n 1}\right| \leqslant\left(E S_{n 1}^{4} E S_{n 1}^{2} \Delta_{n 1}^{2}\right)^{1 / 2}$ 及 $\left|S_{n 1}^{j} \Delta_{n 1}\right| \leqslant\left|\Delta_{n 1}\right|+$ $\left|S_{n 1}^{3} \Delta_{n 1}\right|, j=1,2$ 推得. (16) 式由 $E\left|S_{n 1} \Delta_{n 1}^{3}\right| \leqslant\left(E S_{n 1}^{2} \Delta_{n 1}^{2} E \Delta_{n 1}^{4}\right)^{1 / 2}$ 推得. 引理 3 证毕.

引理 4. 设 $\Lambda_{n *} \subset A_{n}$, 且\# $\left(A_{n *} A_{n}\right) \geqslant \alpha n^{i-\nu}$, 其中 $0 \leqslant \nu<1,0<\alpha \leqslant 1$. 记

$$
S_{n *}=\sum_{(*)} \zeta_{k} / \sqrt{n} \widetilde{B}_{n},
$$

其中 $\sum_{(*)}$ 表示 $\sum_{k \in A_{n *}}$ 则 $\exists \eta, \mu$, 当 $|t| \leqslant \sqrt{n} \eta$ 时有

$$
\left|E\left[S_{n *}^{m} \exp \left(i t s_{n *}\right)\right]\right| \leqslant C\left(1+|t|^{m}\right) \exp \left(-\mu n^{-v} t^{2}\right), \quad m=0,1,2,3 \text {. }
$$

证. 记

$$
\varphi_{k}(t)=\left|E \zeta_{k} \exp \left(i t \zeta_{k} / \sqrt{n} \widetilde{B}_{n}\right)\right|
$$

则

$$
\phi_{k}(t)=\left|E \zeta_{k}\left[\exp \left(i t \zeta_{k} / \sqrt{n} \widetilde{B}_{n}\right)-1\right]\right| \leqslant C n^{-\frac{1}{2}}|t| E \zeta_{k}^{2} .
$$

由引理 2 , 若记 $\mu=\alpha \lambda / 2$, 则当 $|t| \leqslant \sqrt{n} \eta$ 时有

$$
\begin{aligned}
& \left|E \exp \left(i t S_{n *}\right)\right| \leqslant \prod_{k \in A_{n_{*} A_{n}}}\left|\varphi_{n k}\left(t / \sqrt{n} \widetilde{B}_{n}\right)\right| \\
& \leqslant \exp \left(-\frac{\lambda t^{2}}{n} \cdot \frac{\alpha}{2} n^{1-\nu}\right)=\exp \left(-\mu n^{-x} t^{2}\right) \\
& \left|E\left[S_{n *}^{3} \exp \left(i t S_{n *}\right)\right]\right| \leqslant C n^{-3 / 2}\left\{\Sigma_{(*)} E\left|\zeta_{j}\right|^{3} \cdot\left|E \exp \left(i t \sum_{\nu \neq j}(*) \zeta_{v} / \sqrt{n} \widetilde{B}_{n}\right)\right|\right. \\
& +3 \sum_{(*)} E \zeta_{j}^{2} \psi_{k}(t)\left|E \exp \left(i t \sum_{\nu \neq j, k}(*) \zeta_{\nu} / \sqrt{n} \tilde{B}_{n}\right)\right| \\
& \left.+\sum(*) \boldsymbol{\psi}_{i}(t) \boldsymbol{\phi}_{k}(t) \boldsymbol{\psi}_{l}(t) \mid E \exp \left(\text { it } \sum_{\nu \neq i, k, l}(*) \zeta_{v} / \sqrt{n} \widetilde{B}_{n}\right)\right\} \\
& \leqslant C n^{-3 / 2} \exp \left(-\mu n^{-v} t^{2}\right)\left\{\sum_{(*)} E\left|\zeta_{i}\right|^{3}+C n^{-1 / 2}|t| \sum_{(*)} E \zeta_{j}^{2} E \zeta_{k}^{2}\right. \\
& \left.+C n^{-3 / 2}|t|^{3}\left(\sum_{(*)} E \zeta_{j}^{2}\right)^{3}\right\} \\
& \leqslant C n^{-3 / 2} \exp \left(-\mu n^{-v} t^{2}\right)\left(C n+C n^{3 / 2}|t|+C n^{3 / 2}|t|^{3}\right) \\
& \leqslant C\left(1+|t|^{3}\right) \exp \left(-\mu n^{-y} t^{2}\right) \text {. }
\end{aligned}
$$

$m=1$ 和 2 时的证明类似. 引理 4 证毕.

引理 5. $E \exp \left[i t\left(S_{n}-\Delta_{n 1}-\Delta_{n 2}\right)\right] \sim E e^{i t s_{n}}$.

$$
E\left(S_{n}-\Delta_{n 1}-\Delta_{n 2}\right)^{3} \exp \left[i t\left(S_{n}-\Delta_{n 1}-\Delta_{n 2}\right)\right] \sim E\left(S_{n}-\Delta_{n 1}-\Delta_{n 2}\right)^{3} e^{i t S_{n}} \text {. }
$$

证. 由引理 3,4 , 当 $|t| \leqslant \sqrt{n_{\eta}}$ 时有

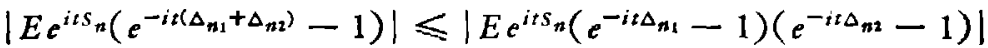

$$
\begin{aligned}
& +\left|E e^{i t s_{n}}\left(e^{-i t \Delta_{n 1}}-1\right)\right|+\left|E e^{i t s_{n}}\left(e^{-i t \Delta_{n 2}}-1\right)\right| \\
& \leqslant t^{2} E\left|\Delta_{n 1}\right| E\left|\Delta_{n 3}\right| \cdot\left|E e^{t t s_{n 3}}\right|+|t| E\left|\Delta_{n 1}\right| \cdot\left|E e^{i t s_{n 2}}\right|+|t| E\left|\Delta_{n 2}\right| \cdot \mid E e^{i t s_{n 1} \mid} \\
& \leqslant C n^{-1} t^{2} \exp \left(-\mu n^{-1 / 8} t^{2}\right)+C n^{-1 / 2}|t| \exp \left(-\mu t^{2}\right) \sim 0 \text {, }
\end{aligned}
$$

这就证明了 (18) 式. 为证 (19) 式,只需证:

$$
\begin{aligned}
& E S_{n}^{m} \Delta_{n 1}^{3-m} e^{i t s_{n}}\left(e^{-i t\left(\Delta_{n 1}+\Delta_{n 2}\right)}-1\right) \sim 0, \quad m-0,1,2,3 . \\
& E \Delta_{n 1}^{2} \Delta_{n 2} e^{i t S_{n}}\left(e^{-i t\left(\Delta_{n 1}+\Delta_{n 2}\right)}-1\right) \sim 0, \\
& E S_{n} \Delta_{n 1} \Delta_{n 2} e^{i t s_{n}}\left(e^{-i t\left(\Delta_{n 1}+\Delta_{n 2}\right)}-1\right) \sim 0 .
\end{aligned}
$$

今证 (20) 式. 以 $m=3$ 为例, 只需证明: 


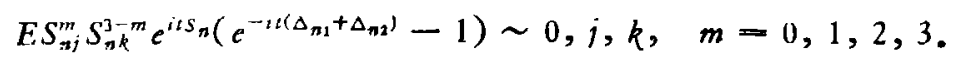

今证

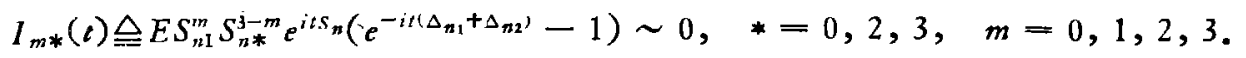

其余证明类似. 由引理 3,4 , 当 $|t| \leqslant \sqrt{n} \eta$ 时,

$$
\begin{aligned}
& \left|I_{m 0}(t)\right| \leqslant E\left|S_{n 0}^{3-m}\right|\left\{\left|E S_{n 1}^{m}\left(e^{-i t \Delta_{n 1}}-1\right)\left(e^{-i t \Delta_{n 2}}-1\right)\right| \cdot\left|E e^{i t S_{n 3}}\right|\right. \\
& \left.+\left|E S_{n 1}^{m}\left(e^{-i t \Delta_{n 1}}-1\right)\right| \cdot\left|E e^{i t s_{n 2}}\right|+\left|E S_{n 1}^{m} e^{-i t S_{n 1}}\right| \cdot\left|E\left(e^{-i t \Delta_{n_{1}}}-1\right)\right|\right\} \\
& \leqslant C t^{2} E\left|S_{n 1}^{m} \Delta_{n 1}\right| \cdot E\left|\Delta_{n 2}\right| \exp \left(-\mu n^{-1 / 8} t^{2}\right) \\
& +C|t| E\left|S_{n 1}^{m} \Delta_{n 1}\right| \exp \left(-\mu t^{2}\right)+C\left(1+|t|^{3}\right) \exp \left(-\mu t^{2}\right)|t| E\left|\Delta_{n 1}\right| \\
& \leqslant C n^{-1} t^{2} \exp \left(-\mu n^{-1 / 8} t^{2}\right)+C n^{-1 / 2}\left(|t|+t^{4}\right) \exp \left(-\mu t^{2}\right) \sim 0 \text {. } \\
& \left|I_{m 2}(t)\right| \leqslant\left|E S_{n 1}^{m} S_{n 2}^{3-m} e^{i t S_{n}}\left(e^{-i t \Delta_{n 1}}-1\right)\left(e^{-i t \Delta_{n 2}}-1\right)\right| \\
& +\sum_{j=1}^{2}\left|E S_{n 1}^{n} S_{n 2}^{3-m} e^{i t S_{n}}\left(e^{-i t \Delta_{n i}}-1\right)\right| \\
& \leqslant t^{2} E\left|S_{n 1}^{m} \Delta_{n 1}\right| \cdot E\left|S_{n 2}^{3-m} \Delta_{n 2}\right| \cdot\left|E e^{i t S_{n 3}}\right| \\
& +|t| E\left|S_{n 1}^{m} \Delta_{n 1}\right| \cdot\left|E S_{n 2}^{3-m} e^{i t S_{n 2} \mid}+\right| t|E| S_{n 2}^{3-m} \Delta_{n 2}|\cdot| E S_{n 1}^{n} e^{i t S_{n 1} \mid} \\
& \leqslant C n^{-1} t^{2} \exp \left(-\mu n^{-1 / 8} t^{2}\right)+C n^{-1 / 2}|t|\left(1+|t|^{3}\right) \exp \left(-\mu t^{2}\right) \sim 0 \text {. }
\end{aligned}
$$

同样有

$$
I_{m 3}(t) \sim 0 .
$$

这就证明了 (23) 式. 类似地可证得(20)中的其它式子. 又, 由引理 3,4 , 当 $|t| \leqslant \sqrt{n} \eta$ 时,

$$
\begin{aligned}
& \left|E \Delta_{n 1}^{2} \Delta_{n 2} e^{i t s_{n}}\left(e^{-i t\left(\Delta_{n 1}+\Delta_{n 2}\right)}-1\right)\right| \\
& \quad \leqslant|t|\left(E\left|\Delta_{n 1}\right|^{3} E\left|\Delta_{n 2}\right|+E \Delta_{n 1}^{2} E \Delta_{n 2}^{2}\right) \mid E e^{i t s_{n 3} \mid} \\
& \quad \leqslant C n^{-3 / 2}|t| \exp \left(-\mu n^{-1 / 8} t^{2}\right) \sim 0,
\end{aligned}
$$

此即 (21) 式. 同样可证得 (22) 式. 因而得到 (19) 式.

引理 6. 令

$$
\begin{aligned}
\pi_{n}(t)= & i^{3} E S_{n}^{3} e^{i t S_{n}}+i^{3}\left[-3 E S_{n}^{2}\left(\Delta_{n 1}+\Delta_{n 2}\right)+3 E S_{n}\left(\Delta_{n 1}+\Delta_{n 2}\right)^{2}\right. \\
& \left.-E\left(\Delta_{n 1}+\Delta_{n 2}\right)^{3}\right] e^{-t^{2} / 2}
\end{aligned}
$$

则有

$$
i^{3} E\left(S_{n}-\Delta_{n 1}-\Delta_{n 2}\right)^{3} e^{i t s_{n}} \sim \pi_{n}(t) .
$$

证. 为证 (24) 式, 只须证明对满足 $v_{0} \leqslant 2, v_{0}+v_{1}+v_{2}=3$ 的非负整数 $v_{0}, v_{1}, v_{2}$, 有

$$
E S_{n}^{\nu_{0}} \Delta_{n_{1}}^{\nu_{1}} \Delta_{n_{2}}^{\nu_{2}} e^{i t S_{n}} \sim E S_{n}^{\nu_{0}} \Delta_{n_{1}}^{\nu_{1}} \Delta_{n_{2}}^{\nu_{2}} e^{-t^{2} / 2}
$$

各式证明类似,以 $\left(v_{0}, v_{1}, v_{2}\right)=(2,1,0)$ 为例, 即要证:

$$
E S_{n}^{2} \Delta_{n 1} e^{i t s_{n}} \sim E S_{n}^{2} \Delta_{n 1} e^{-t^{2} / 2} \text {. }
$$

不失普遍性, 可设 $\Delta_{n 1}=\frac{1}{\sqrt{n} \widetilde{B}_{n}} \sum_{(1)} a_{j} a_{k} e_{j} e_{k}$, 其中, $\sum_{(1)} a_{k}^{2} \leqslant 1$. 记 $Z_{k}-a_{k} e_{k}, \vartheta_{k}(t)=$ $\left|E Z_{k} \exp \left(i t \zeta_{k} / \sqrt{n} \tilde{B}_{n}\right)\right|, \phi_{k}(t)=\left|E \zeta_{k} \exp \left(i t \zeta_{k} / \sqrt{n} \widetilde{B}_{n}\right)\right|$, 由引理 4 证明的开头, 有 $\psi_{k}(t) \leqslant$ $C n^{-1 / 2}|t| E \zeta_{k}^{2}$, 同样, $\vartheta_{k}(t) \leqslant C n^{-1 / 2}|t| E\left|Z_{k} \zeta_{k}\right|$. 由引理 4, 并考虑到 (17) 式, 当 $|t| \leqslant \sqrt{n_{\eta}}$ 时有 


$$
\begin{aligned}
& \left|E S_{n}^{2} \Delta_{n 1} e^{t t s_{n}}-2 n^{-3 / 2} \widetilde{B}_{n}^{-3} \sum_{j \neq k} E\left(\zeta_{j} Z_{i} \zeta_{k} Z_{k} e^{r t S_{n}}\right)\right| \\
\leqslant & C n^{-3 / 2}\left\{\sum_{j \neq k \neq t} \sum_{(1)} E \zeta_{j}^{2} \vartheta_{k}(t) \vartheta_{l}(t) e^{-\mu t^{2}}+\sum_{j \neq k} E\left|\zeta_{j}^{2} Z_{j}\right| \vartheta_{k}(t) e^{-\mu t^{2}}\right. \\
& \left.+\sum_{i \neq k \neq l} \sum_{(1)} \psi,(t) E\left|\zeta_{k} Z_{k}\right| \vartheta_{l}(t) e^{-\mu t^{2}}+\sum_{i \neq k+\neq l \neq m} \sum_{(t)} \psi_{j}(t) \psi_{k}(t) \vartheta_{l}(t) \vartheta_{m}(t) e^{-\mu t^{2}}\right\} \\
\leqslant & C n^{-5 / 2} t^{2} \sum E \zeta_{j}^{2}\left(\sum_{(1)} E\left|Z_{j} \zeta_{j}\right|\right)^{2} e^{-\mu t^{2}}+C n^{-2} \cdot|t| \sum_{(1)} E\left|\zeta_{j}^{2} Z_{j}\right| \sum_{(1)} E\left|Z_{i} \zeta_{j}\right| e^{-\mu t^{2}} \\
& +C n^{-5 / 2} t^{2} \sum E \zeta_{j}^{2}\left(\sum_{(1)} E\left|Z_{j} \zeta_{j}\right|\right)^{2} e^{-\mu t^{2}} \\
& +C n^{-7 / 2} t^{4}\left(\sum E \zeta_{j}^{2}\right)^{2}\left(\sum_{(1)} E\left|Z_{j} \zeta_{j}\right|\right)^{2} e^{-\mu t^{2}} \\
\leqslant & C n^{-1 / 2}\left(|t|+t^{4}\right) e^{-\mu t^{2}} \sim 0,
\end{aligned}
$$

故

$$
E S_{n}^{2} \Delta_{n 1} e^{i s S_{n}} \sim 2 n^{-3 / 2} \widetilde{B}_{n}^{-3} \sum_{j \neq k} E\left(\zeta_{j} Z_{j} \zeta_{k} Z_{k} e^{i t s_{n}}\right) .
$$

由文献 [4]，p. 109，引理 1, 在定理条件下, $\exists \eta$, 使

$$
\left|E e^{i t s_{n}}-e^{-t^{2 / 2}}\right| \leqslant C n^{-1 / 2}|t|^{3} e^{-t^{2 / 3}} \text {, 当 }|t| \leqslant \sqrt{n} \eta \text {. }
$$

不妨设 $\mu \leqslant 1 / 3$. 此式与引理 4 相结合, 并考虑到 $(17)$ 式, 则当 $|t| \leqslant \sqrt{n} \eta$ 时有

$$
\begin{aligned}
& 2 n^{-3 / 2} \tilde{B}_{n}^{-3} \mid \sum_{(1)} E\left(\zeta_{i} Z_{j} \zeta_{k} Z_{k} e^{\left.i t S_{n}\right)}-\sum_{(1)} E\left(\zeta_{j} Z_{j} \zeta_{k} Z_{k}\right) e^{-t^{2} / 2} \mid\right. \\
\leqslant & C n^{-3 / 2} \sum_{(1)} E\left|\left(\zeta_{j} Z_{i} \zeta_{k} Z_{k}-E \zeta_{j} Z_{j} \zeta_{k} Z_{k}\right)\left[\exp \left(i t\left(\zeta_{j}+\zeta_{k}\right) / \sqrt{n} \tilde{B}_{n}\right)-1\right]\right| \\
& \times\left|E \exp \left(i t \sum_{v \neq j, k} \zeta_{v} / \sqrt{n} \tilde{B}_{n}\right)\right|+C n^{-3 / 2} \sum_{(1)}\left|E \zeta_{j} Z_{j} E \zeta_{k} Z_{k}\right| \\
& \times \mid E e^{i t S_{n}}-e^{-t^{2 / 2} \mid} \\
\leqslant & C n^{-2}|t| \sum_{(1)} E\left|\zeta_{j} Z_{j} \zeta_{k} Z_{k}-E \zeta_{j} Z_{i} \zeta_{k} Z_{k}\right|\left(\left|\zeta_{j}\right|+\left|\zeta_{k}\right|\right) e^{-\mu t^{2}} \\
& +C n^{-2}|t|^{3}\left(\sum_{(1)} E\left|Z_{j} \zeta_{j}\right|\right)^{2} e^{-t^{2} / 3} \\
\leqslant & C n^{-2}|t| \sum_{(1)} E\left|\zeta_{j} Z_{j} \zeta_{k} Z_{k}\right|\left(\left|\zeta_{j}\right|+\left|\zeta_{k}\right|\right) e^{-\mu t^{2}}+C n^{-2}|t|^{3} \cdot C n e^{-\mu k^{*}} \\
\leqslant & C n^{-2}|t| \sum_{(1)} E\left|\zeta_{j}^{2} Z_{j}\right| \sum_{(1)} E\left|\zeta_{j} Z_{j}\right| e^{-\mu t^{2}}+C n^{-1}|t|^{3} e^{-\mu t^{2}} \\
\leqslant & C n^{-2}|t| \cdot C n \cdot C n^{1 / 2} e^{-\mu t^{2}}+C n^{-1}|t|^{3} e^{-\mu t^{2}} \\
\leqslant & C n^{-1 / 2}\left(|t|+|t|^{3}\right) e^{-\mu t^{2}} \sim 0,
\end{aligned}
$$

故

$$
E S_{n}^{2} \Delta_{n 1} e^{i t S_{n}} \sim 2 n^{-3 / 2} \widetilde{B}_{n}^{-3} \Sigma_{(1)} E \zeta_{j} Z_{j} E \zeta_{k} Z_{k} e^{-t^{2 / 2}},
$$

这就证明了 $\left(25^{\prime}\right)$. 其余各式可类似地证得.

引理 7. 设 $\varphi(t)$ 在 $|t| \leqslant T$ 内有三阶连续导函数 $\varphi^{(3)}(t)$, 且 $\varphi^{(m)}(0)-0, m=0$, 1,2 , 则

$$
\int_{|z|<T}|t|^{m-4}\left|\varphi^{(m)}(t)\right| d t \leqslant \int_{|t|<T}|t|^{-1}\left|\varphi^{(3)}(t)\right| d t, \quad m=0,1,2 .
$$

证. 见文献 [1], 引理 2 .

引理 8. 设 $G_{n}(x)$ 非降, $H_{n}(x)$ 为有界变差函数, 其 F.S. 变换分别为 $g_{n}, h_{n}$. 设

$$
G_{n}( \pm \infty)=H_{n}( \pm \infty), \quad \int_{-\infty}^{\infty}|x|^{3}\left|d\left(G_{n}(x)-H_{n}(x)\right)\right|<\infty,
$$


且

$$
\left|H_{n}(x)-\Phi(x)\right| \leqslant C /\left[\sqrt{n}(1+|x|)^{3}\right]
$$

则对 $T_{n}=\sqrt{n} \eta$, 有

$$
\begin{aligned}
\left|G_{n}(x)-H_{n}(x)\right| \leqslant & C(1+|x|)^{-3}\left\{\int_{|\ell|<T_{n}}|t|^{-1}\left|g_{n}(t)-h_{n}(t)\right| d t\right. \\
& \left.+\int_{|t| \leqslant T_{n}}|t|^{-1}\left|\delta_{3}^{(n)}(t)\right| d t+C / \sqrt{n}\right\}
\end{aligned}
$$

其中，

$$
\delta_{3}^{(n)}(t)=\int_{-\infty}^{\infty} e^{i t x} d\left\{x^{3}\left(G_{n}(x)-H_{n}(x)\right)\right\}
$$

证. 见文献 [5].

引理 9. 设 $W_{n}=a_{n} W_{n 1}+W_{n 2}+b_{n}, n=1,2, \cdots, W_{n 1}, W_{n 2}$ 为 r. v., $a_{n}, b_{n}$ 为实 数. $W_{n}, W_{n 1}$ 的分布分别为 $G_{n}, G_{n 1}$. 设当 $n$ 充分大时,

$$
\begin{gathered}
\left|G_{n 1}(x)-\Phi(x)\right| \leqslant C n^{-1 / 2}(1+|x|)^{-3}, \\
P\left(\left|W_{n 2}\right| \geqslant C|x| / \sqrt{n}\right) \leqslant C n^{-1 / 2}(1+|x|)^{-3}, \text { 当 }|x| \geqslant 1, \\
\left|a_{n}-1\right| \leqslant C / \sqrt{n}, \quad\left|b_{n}\right| \leqslant C / \sqrt{n},
\end{gathered}
$$

则当 $n$ 充分大时,有

$$
\left|G_{n}(x)-\Phi(x)\right| \leqslant C n^{-1 / 2}(1+|x|)^{-3} .
$$

证. 见文献 [1], 引理 1 .

\section{三、定 理 证 明}

记 $a_{2}=E\left(\Delta_{n 1}+\Delta_{n 2}\right)^{2}, a_{3}=-3 E S_{n}^{2}\left(\Delta_{n 1}+\Delta_{n 2}\right)+3 E S_{n}\left(\Delta_{n 1}+\Delta_{n 2}\right)^{2}-E\left(\Delta_{n 1}+\Delta_{n 2}\right)^{3}$. 易见

$$
\left|a_{k}\right| \leqslant C / \sqrt{n}, \quad k=2,3 \text {. }
$$

定义

$$
\begin{aligned}
& h_{n}(t)=E e^{i t s_{n}}+\sum_{k=2}^{3} \frac{a_{k}(i t)^{k}}{k !} e^{-t^{2} / 2}, \\
& g_{n}(t)=E \exp \left\{i t\left(S_{n}-\Delta_{n 1}-\Delta_{n 2}\right)\right\} .
\end{aligned}
$$

由（34）式易见，

$$
\left|h_{n}^{(3)}(t)-\pi_{n}(t)\right| \leqslant C_{n}^{-1 / 2}\left(|t|+t^{6}\right) e^{-t^{2 / 2}}
$$

$\pi_{n}(t)$ 的定义见引理 6. 故由引理 5,6 , 有

$$
g_{n}^{(3)}(t) \sim \pi_{n}(t) \sim h_{n}^{(3)}(t),
$$

即 $\exists \eta$, 使

$$
\int_{|z|<\sqrt{n} \eta}|t|^{-1}\left|g_{n}^{(3)}(t)-h_{n}^{(3)}(t)\right| d t \leqslant C / \sqrt{n} .
$$

又因 $g_{n}^{(m)}(0)=h_{n}^{(m)}(0), m=0,1,2$. 故由引理 7, 有

$$
\int_{|z|<\sqrt{n} \eta}|t|^{m-4}\left|g_{n}^{(m)}(t)-h_{n}^{(m)}(t)\right| d t \leqslant C / \sqrt{n}, \quad m=0,1,2 .
$$


由 (34). (35) 式及引理 5,

$$
h_{n}(t) \sim E e^{i t s_{n}} \sim g_{n}(t),
$$

即

$$
\int_{|\ell| \leqslant \sqrt{n} \eta}|t|^{-1}\left|g_{n}(t)-h_{n}(t)\right| d t \leqslant C / \sqrt{n} .
$$

令

$$
\begin{gathered}
H_{n}(x)=P\left(S_{n} \leqslant x\right)+\sum_{k=2}^{3} \frac{(-1)^{k} a_{k}}{k !} \Phi^{(k)}(x), \\
G_{n}(x)=P\left(S_{n}-\Delta_{n 1}-\Delta_{n 2} \leqslant x\right),
\end{gathered}
$$

由独泣和之已知结果 (见文献 $[4]$, 第五章 $\$ 5$ ),

$$
\left|P\left(S_{n} \leqslant x\right)-\Phi(x)\right| \leqslant C n^{-1 / 2}(1+|x|)^{-3},
$$

故由（34）及(40) 式,有

$$
\left|H_{n}(x)-\Phi(x)\right| \leqslant C n^{-1 / 2}(1+|x|)^{-3} .
$$

易见、 $G_{n}(x)$ 及 $H_{n}(x)$ 满足引理 8 的所有条件, 故

$$
\begin{aligned}
\left|G_{n}(x)-H_{n}(x)\right| \leqslant & C(1+|x|)^{-3}\left\{\int_{|t|<\sqrt{n} \eta}|t|^{-1}\left|g_{n}(t)-h_{n}(t)\right| d t\right. \\
& \left.+\int_{|t| \leqslant \sqrt{n} \eta}|t|^{-1}\left|\delta_{3}^{(n)}(t)\right| d t+C / \sqrt{n}\right\},
\end{aligned}
$$

其中，

$$
\delta_{3}^{(n)}(t)=\int_{-\infty}^{\infty} e^{i t x} d\left\{x^{3}\left(G_{n}(x)-H_{n}(x)\right)\right\} .
$$

由文献 [4], p. 153, 引理 7, 并注意到 (37)一-(39) 式及 (44) 式,有

$$
\begin{aligned}
\left|G_{n}(x)-H_{n}(x)\right| \leqslant & C(1+|x|)^{-3}\left\{\int_{|t|<\sqrt{n} \eta}|t|^{-1}\left|g_{n}(t)-h_{n}(t)\right| d t\right. \\
& \left.\quad+\sum_{m=0}^{3} \int_{|t|<\sqrt{\pi} \eta}|t|^{m-4}\left|g_{n}^{(m)}(t)-h_{n}^{(m)}(t)\right| d t+C / \sqrt{n}\right\} \\
\leqslant & C n^{-1 / 2}(1+|x|)^{-3} .
\end{aligned}
$$

再由 (43) 式,我们有

$$
\left|G_{n}(x)-\Phi(x)\right| \leqslant C_{n^{-1 / 2}}(1+|x|)^{-3}
$$

由引理 3 , 对 $|x| \geqslant 1$,

$$
\begin{aligned}
P\left(\left|\Delta_{n 3}\right| \geqslant|x| / \sqrt{n}\right) & \leqslant n^{2} x^{-4} E\left|\Delta_{n 3}\right|^{4} \leqslant n^{2} x^{-4} \cdot C n^{-5 / 2} \\
& \leqslant C n^{-1 / 2}(1+|x|)^{-3}
\end{aligned}
$$

记 $V_{n}-S_{n}-\Delta_{n 1}-\Delta_{n 2}-\Delta_{n 3}$, 则由引理 9,

$$
\left|P\left(V_{n} \leqslant x\right)-\Phi(x)\right| \leqslant C n^{-1 / 2}(1+|x|)^{-3} .
$$

命

$$
Q_{n}^{(1)}=\frac{1}{\sqrt{n} \widetilde{B}_{n}} \sum_{k=1}^{n}\left(e_{k}^{2}-\sigma^{2}\right)-\frac{1}{\sqrt{n} \widetilde{B}_{n}} \sum_{j=1}^{r}\left(\sum_{k=1}^{n} a_{n i k} e_{k}\right)^{2},
$$

因 $\left(\sum_{k} a_{n j k} e_{k}\right)^{2} \leqslant \sum_{k} a_{n i k}^{2} \sum_{(0)} e_{k}^{2} \leqslant \sum_{(0)} e_{k}^{2}$, 故 


$$
\begin{aligned}
\frac{1}{\sqrt{n} \widetilde{B}_{n}} \sum_{j=1}^{r}\left(\sum_{k=1}^{n} a_{n j k} e_{k}\right)^{2} & \leqslant \sum_{*=0}^{3} \frac{4}{\sqrt{n} \widetilde{B}_{n}} \sum_{j=1}^{r}\left(\sum_{\left.(*) a_{n j k} e_{k}\right)^{2}}\right. \\
& \leqslant \frac{1}{\sqrt{n} \tilde{B}_{n}}\left(\sum_{(0)} 4 r e_{k}^{2}+\sum^{\prime} 4 \lambda_{k} e_{k}^{2}\right)+\Delta_{n 1}+\Delta_{n 2}+\Delta_{n 3},
\end{aligned}
$$

又因 $1-4 \lambda_{k} \geqslant 0$ 当 $k \in \Lambda_{n}-\Lambda_{n 0}$, 故 $\Sigma^{\prime}\left(1-4 \lambda_{k}\right) e_{k}^{2} \geqslant \Sigma^{\prime}\left(1-4 \lambda_{k}\right) \hat{e}_{k}^{2}$, 因此,

$$
\begin{aligned}
Q_{n}^{(1)} \geqslant & \frac{1}{\sqrt{n} \widetilde{B}_{n}}\left\{\sum_{(0)}(1-4 r)\left(e_{k}^{2}-\sigma^{2}\right)-(4 r)^{2} \sigma^{2},\right. \\
& \left.+\Sigma^{\prime}\left(1-4 \lambda_{k}\right) e_{k}^{2}-\Sigma^{\prime} \sigma^{2}\right\}-\Delta_{n 1}-\Delta_{n 2}-\Delta_{n 3} \\
\geqslant & \frac{1}{\sqrt{n} \tilde{B}_{n}} \sum_{k=1}^{n} \zeta_{k}-\Delta_{n 1}-\Delta_{n 2}-\Delta_{n 3}-\dot{u}_{n}=V_{n}-u_{n},
\end{aligned}
$$

其中，

$$
\begin{aligned}
u_{n} & =\frac{1}{\sqrt{n} \widetilde{B}_{n}}\left\{(4 r)^{2} \sigma^{2}+\Sigma^{\prime}\left(\sigma^{2}-E \hat{e}_{k}^{2}\right)+4 \Sigma^{\prime} \lambda_{k} E \hat{e}_{k}^{2}\right\} \\
& \leqslant C n^{-1 / 2}\left\{C+\Sigma^{\prime} E e_{k}^{2} I\left(e_{k}^{2}>\sqrt{n}\right)+C\right\} \\
& \leqslant C n^{-1 / 2}\left\{C+\frac{1}{n} \Sigma^{\prime} E e_{k}^{6}+C\right\} \leqslant c n^{-1 / 2},
\end{aligned}
$$

故由（49）式,有

$$
\left|P\left(V_{n}-u_{n} \leqslant x\right)-\Phi(x)\right| \leqslant C_{n^{-1 / 2}}(1+|x|)^{-3}
$$

命

$$
\begin{gathered}
T_{n}=\frac{1}{\sqrt{n} B_{n}} \sum\left(e_{k}^{2}-\sigma^{2}\right), \\
\widetilde{T}_{n}=\frac{1}{\sqrt{n} \widetilde{B}_{n}} \sum\left(e_{k}^{2}-\sigma^{2}\right)=\frac{B_{n}}{\widetilde{B}_{n}} T_{n},
\end{gathered}
$$

则由独立和之已知结果，

$$
\left|P\left(T_{n} \leqslant x\right)-\Phi(x)\right| \leqslant C_{n^{-1 / 2}}(1+|x|)^{-3},
$$

但因 $\left|B_{n} / \widetilde{B}_{n}-1\right| \leqslant C / \sqrt{n}$, 故由引理 9 ,

$$
\left|P\left(\widetilde{T}_{n} \leqslant x\right)-\Phi(x)\right| \leqslant C n^{-1 / 2}(1+|x|)^{-3} \text {. }
$$

但

$$
V_{n}-u_{n} \leqslant Q_{n}^{(1)} \leqslant \widetilde{T}_{n}
$$

故由（54）及（56）式，

$$
\left|P\left(Q_{n}^{(1)} \leqslant x\right)-\Phi(x)\right| \leqslant C n^{-1 / 2}(1+|x|)^{-3} .
$$

命

$$
Q_{n}^{(2)}=\frac{1}{\sqrt{n} B_{n}} \sum_{k=1}^{n}\left(e_{k}^{2}-\sigma^{2}\right)-\frac{1}{\sqrt{n} B_{n}} \sum_{j=1}^{r}\left(\sum_{k=1}^{n} a_{n / k} e_{k}\right)^{2}=\frac{\widetilde{B}_{n}}{B_{n}} Q_{n}^{(1)},
$$

则由 $\left|\widetilde{B}_{n} / B_{n}-1\right| \leqslant C / \sqrt{n}$ 及引理 9,

$$
\left|P\left(Q_{n}^{(2)} \leqslant x\right)-\Phi(x)\right| \leqslant C n^{-1 / 2}(1+|x|)^{-3} .
$$

最后，按照本文开头的记号， 


$$
Q_{n}=\left(\hat{\sigma}_{n}^{2}-\sigma^{2}\right) / \sqrt{\operatorname{Var}\left(\hat{\sigma}_{n}^{2}\right)}=\frac{\sqrt{n} B_{n}}{(n-r) \sqrt{\operatorname{Var}\left(\hat{\sigma}_{n}^{2}\right)}} Q_{n}^{(2)},
$$

其分布函数已记为 $F_{n}$. 据文献 [3], 引理 8 , 有

$$
\left|\frac{\sqrt{n} B_{n}}{(n-r) \sqrt{\operatorname{Var}\left(\hat{\sigma}_{n}^{2}\right)}}-1\right| \leqslant c / \sqrt{n},
$$

故由 (60)-(62) 式及引理 9, 即得 (4) 式. 定理 1 证毕.

致谢. 本文得到陈希孺教授的指导，谨致以衰心的感谢。

\section{* 考文献}

[1] 赵林域、陈希诫,中国科学 A 辑, 1982,5: 408 .

[2] 苏序,科学通报, 28(1983), 12: 705 .

[ 3 ] 陈希㩧,中国科学, 1981，2: 129.

[4] Petrov, V. V., Sums of Independent Random Variables, Springer-Verlag, 1975.

[5] 赵林城、陈希嘴,中国科学 A 辑, 1982,12: 1066. 